\title{
Tendencias de investigación en los posgrados de gestión educativa en América Latina
}

\section{María Paola Bertel-Narváez}

orcid.org/0000-0003-0579-3102

Universidad del Magdalena, Colombia.

mbertel@unimagdalena.edu.co

\section{Jorge Oswaldo Sánchez-Buitrago}

orcid.org/00oo-0oo2-9299-6647

Universidad del Magdalena, Colombia.

joswaldosanchez@unimagdalena.edu.co
Javier de Jesús Viloria-Escobar

orcid.org/00oo-0002-2396-4190

Universidad del Magdalena, Colombia.

jviloriae@unimagdalena.edu.co

\section{Resumen}

Este artículo muestra las tendencias investigativas en las maestrías y doctorados en el área de la gestión educativa, en América Latina, a partir de la revisión de las líneas de investigación de las doce mejores universidades en 2017, según el ranking Quacquarelli Symonds (OS). Los resultados evidencian como tendencia principal los procesos de gestión académica, seguidos por la gestión comunitaria y directiva; y, por último, los relacionados con la gestión administrativa y financiera.

\section{Palabras claves (Fuente: Tesauro de la Unesco)}

Educación superior; educación posgradual; investigación en la universidad; gestión educativa; América Latina.

Recepción: 23/08/2018 | Envío a pares: 09/02/2019 | Aceptación por pares: 18/03/2019 | Aprobación: 13/05/2019 


\title{
Research Trends in Educational Management Graduate Programs in Latin America
}

\begin{abstract}
This article shows research trends in master's and Ph.D. programs in educational management in Latin America by reviewing the research lines of the top twelve universities in 2017, according to the Quacquarelli Symonds (OS) Ranking. Results reveal academic management as the main trend, followed by community and executive management and, lastly, administrative and financial management.
\end{abstract}

Keywords (Source: Unesco Thesaurus)

Higher education; university education; postgraduate education; postgraduate courses; university research; academic management; educational management; Latin America. 


\section{Tendências de pesquisa nas pós-graduações de gestão educativa na América Latina}

Resumo

Este artigo mostra as tendências de pesquisa em mestrados e doutorados na área de gestão educativa na América Latina, a partir da revisão das linhas de pesquisa das doze melhores universidades em 2017, segundo o ranking Quacquarelli Symonds (QS). Os resultados tornam evidentes, como tendência principal, os processos de gestão acadêmica, seguidos pela gestão comunitária e diretiva, e, por último, os de gestão administrativa e financeira.

Palavras-chave (Fonte: tesauro da Unesco)

Educação superior; ensino superior; educação de pós-graduação; curso pós-universitário; pesquisa universitária; gestão educacional; América Latina. 


\section{Introducción}

Históricamente se ha sostenido la premisa que considera a la educación como la herramienta más pertinente para la transformación de las realidades sociales y el desarrollo de los territorios (Díaz y Alemán, 2008; Botero, 2009; Ardashkin, 2015; Dumciuviene, 2015; Kangjuan, Anyu, Siyi, Maoguo y Xiaohong, 2017). Por ello, la gestión de las organizaciones educativas es un asunto clave para lograr las intencionalidades teleológicas que subyacen en estas, es decir, la formación humana (Gamage, 2006; Connolly, James y Fertig, 2017). La gestión de las organizaciones educativas es una tarea que requiere de directivos con competencias teóricas, metodológicas e investigativas para el ejercicio de sus funciones de direccionamiento (Sánchez, Sánchez y Viloria, 2016; 2017). En este terreno, el presente trabajo expone las dinámicas y orientaciones investigativas en el campo de la gestión educativa de las instituciones de educación superior de América Latina mejor posicionadas en el ámbito educativo, de acuerdo con el ranking Quacquarelli Symonds (OS).

Los resultados de la investigación tipifican las líneas de investigación de acuerdo con los lineamientos de la Guía para el Mejoramiento Institucional número 34 del Ministerio de Educación Nacional de Colombia, la cual describe las cuatro áreas que integran la gestión de las instituciones educativas: gestión directiva, gestión académica, gestión administrativa y financiera, y gestión de la comunidad. Se toma como referencia esta Guía por la necesidad de aportar a los procesos de mejoramiento en el contexto nacional a través de la formación investigativa de gestores educativos que reconozcan las tendencias latinoamericanas y las incorporen en los procesos de gestión de las organizaciones educativas en el territorio colombiano. En este sentido, teniendo una lectura continental que parte de los lineamientos del país, se pueden promover transformaciones y resignificaciones en el sector educativo.

Esta tificación de líneas de investigación en el campo de la gestión educativa es un producto académico generado en el marco de la línea de investigación "Administración y desarrollo de los sistemas educativos", privilegiada en el Grupo de Investigación en Gestión Pedagógica Transformadora (GEPET), de la Universidad del Magdalena. Esta línea de investigación se originó al conjugar intereses epistémicos de las ciencias de la Educación y de las ciencias de la Administración. De allí nace la pretensión investigativa, académica y social de concebir a la gestión educativa no exclusivamente desde la óptica de lo administrativo, sino también como una dinámica que debe traer inmerso transversalmente lo pedagógico, es decir, la formación humana. Es así como este proceso investigativo se convierte también en una posibilidad para las orientaciones académicas de la Línea.

Se considera que las líneas de investigación se constituyen en el instrumento o herramienta básica necesaria para "la planeación de las actividades de investigación en el ámbito institucional" (Cerda, 2004, p. 21). Por lo tanto, es en las líneas de investigación donde se evidencian, en primera instancia, las intenciones investigativas de los grupos de investigación y/o programas de formación avanzada, y es así como los investigadores recurren a las líneas de investigación existentes para dilucidar los trayectos investigativos que se han transitado en el campo de la gestión educativa. Sánchez (2012) establece que la naturaleza de las líneas de investigación en el entorno institucional de los programas académicos o en las organizaciones educativas no puede desligarse de la organización del trabajo investigativo; por lo cual, estas son concebidas por aquellos sujetos a quienes les concierne implementar políticas o procesos investigativos que integran determinado sistema.

Para lograr una mejor comprensión del concepto de línea de investigación, se adopta la teorización expuesta por Cerda, quien asevera que una línea de investigación puede ser comparada con una línea geométrica:

Todos sabemos que en geometría la línea es una sucesión continua de puntos en el espacio y en 
general se hace referencia a un trazo o una extensión continua que se considera o se percibe sólo en su longitud. Precisamente este carácter longitudinal es el que le da identidad a una linea que se mueve o se desarrolla en el sentido de la longitud. Al igual que las líneas de investigación, que nos indican el curso y dirección de un conjunto de temas, problemas y contenidos permanentes en torno al cual se organizan sistemáticamente los proyectos de investigación que se llevan a cabo. Muy similar a una linea vectorial que tiene magnitud, sentido, dirección, fuerza, coherencia y un punto de aplicación. (2000, p. 21)

La línea de investigación emerge como el soporte sobre el cual se planifica y ejerce la labor investigativa de uno o más individuos, equipos e instituciones, y si todo ello se fundamenta de manera racional, propicia la integración efectiva de sus partes constituyentes, bajo la premisa fundante del desarrollo epistémico, empírico y/o metodológico en un área específica del saber (Barrios, 1990). De esta manera, las líneas de investigación en gestión educativa tienen como propósito concretar esfuerzos investigativos de forma sistemática y ordenada a través del trabajo conjunto de los miembros de un grupo de investigación o de los desarrollos investigativos alcanzados en un programa de formación avanzada.

La gestión educativa está definida como la disciplina cuyo objeto es "el estudio de la organización del trabajo en el campo de la educación. Por lo tanto, está determinada por el desarrollo de las teorías generales de la gestión y los de la educación. Pero no se trata de una disciplina teórica. Su contenido disciplinario está determinado tanto por los contenidos de la gestión como por la cotidianidad de su práctica" (Casassus, 2000, p. 49).

Sin embargo, en su naturaleza, la gestión educativa no puede ser comprendida como una simple práctica en la que se conjuga lo administrativo y lo educativo (Vidal, Durán y Pujal, 2008; Farfán, Mero y Sáenz, 2016). Para lograr una verdadera comprensión de esta categoría es imperativo entenderla como una praxis capaz de resignificarse y de adoptar sus propios sentidos y significados, todo ello posibilitado por las mediaciones pedagógicas que tienen lugar en las organizaciones educativas, no solo dentro del aula de clases, sino de manera transversal en todas las áreas o procesos que integran la gestión de las organizaciones educativas (Rodríguez, 2017a/b; González, 2017). Es así como se entiende la organización educativa como un sistema pedagógico en el que confluyen distintos actores que configuran las realidades educativas del territorio.

Es de destacar que el paradigma de la complejidad no es ajeno a las mencionadas realidades educativas, por lo que la comprensión efectiva de estas se hace cada vez más desafiante. Para ello, la formación avanzada de calidad emerge como estrategia fundamental que propicia la adquisición de competencias de gestión necesarias por parte de los actores educativos, especialmente de los docentes y directivos docentes. Es preponderante para las organizaciones y los sistemas educativos contar con mediaciones investigativas y metodológicas que aporten a la mejor comprensión de las mencionadas realidades y que posibiliten la transformación de estas (Tejada, 2000; Castro, 2001; López, 2009; Hernández, 2016).

En Colombia la formación avanzada de docentes y directivos docentes está encaminada a la puesta en marcha de procesos investigativos, de innovación y gestión educativa, todo ello fundamentado en la reflexión crítica sobre los entornos educativos, a nivel tanto micro (problemáticas y retos locales) como macro (problemáticas y retos del sistema educativo) (MEN, 2013). Ello se debe a que "el objetivo de la educación postgradual en las universidades se puede definir como la realización de investigaciones que contribuirían al desarrollo de la ciencia, diseminando dicha investigación en el público y satisfaciendo las necesidades del profesor en los dominios requeridos" (Başarı, Aktepebaşı, Yağcı y Akdağ, 2017, p. 888). 
La evaluación de la pertinencia y calidad de los procesos de formación avanzada en el país está directamente relacionada con la calidad de los programas de posgrado, especialmente de las maestrías investigativas y los doctorados, las cuales articulan su gestión investigativa a través de las líneas de investigación, las que, por efecto, deben responder a las realidades del territorio, siendo focalizadoras de dinámicas académicas e investigativas que aporten comprensiones que puedan traducirse en transformaciones educativas pertinentes. Para ello el aseguramiento de la calidad de los programas postgraduales es "un proceso de importancia estratégica, por cuanto el currículo de este nivel educativo debe ser el más transformador" (Morillo y González, 2016, p. 138).

Este artículo toma especial importancia por sus aportes a la comprensión de las tendencias investigativas en el campo de la gestión educativa en programas de maestría y doctorado de América Latina, como punto de partida para la configuración de escenarios pertinentes de formación avanzada para directivos y docentes en Colombia y, en consecuencia, el mejoramiento de los procesos de gestión de las organizaciones educativas en el país.

\section{Metodología}

En relación con el procesamiento de la información, los investigadores realizaron una tipificación de las líneas de investigación de los programas de formación avanzada de las 12 universidades latinoamericanas mejor posicionadas en el ranking QS

1 El ranking mundial de universidades OS es una clasificación que se realiza cada año de aproximadamente 800 Universidades de todo el mundo, publicado por Quacquarelli Symonds, una compañía británica especializada en educación superior. Aunque el ranking muestra los desempeños globales de las distintas universidades, también presenta clasificaciones a nivel regional, por área de estudio, por facultad, según fortaleza del sistema educativo, entre otros enfoques. Los criterios para la clasificación de las universidades tienen en cuenta seis elementos: reputación académica (40\%), reputación del empleador (10\%), relación estudiante-facultad (20\%), citaciones por facultad (20\%), relación de catedráticos internacionales (5\%) y estudiantes internacionales (5\%). en el área de Educación y Formación, en el año 2017, para posteriormente develar las tendencias investigativas en América Latina, en lo correspondiente a las cuatro áreas de la gestión educativa propuestas por el Ministerio de Educación Nacional en su Guía para el Mejoramiento Institucional. Para ello, se exploraron los programas de formación avanzada de las distintas facultades de Educación de las mencionadas universidades y se analizaron de manera crítica sus líneas de investigación. Las 12 universidades mejor posicionadas en el campo de la educación en América Latina en el 2017 fueron:

1. Pontificia Universidad Católica de Chile.

2. Universidad Nacional Autónoma de México (UNAM).

3. Universidad de Chile.

4. Universidad de Buenos Aires (UBA).

5. Universidade de São Paulo.

6. Universidade Estadual de Campinas (Unicamp).

7. Universidad Nacional de Colombia.

8. Pontificia Universidad Católica de Valparaíso.

9. Universidad de los Andes.

10. Universidad de Santiago de Chile (USACH).

11. Universidade Federal do Rio de Janeiro.

12. Universidade Federal do Rio Grande Do Sul.

La información fue recolectada de los sitios web de las universidades y sus respectivos programas de posgrado. En primera instancia, ella fue sistematizada en una matriz por universidad teniendo en cuenta la información proporcionada por cada institución, donde se relacionan los programas, las líneas de investigación y su descripción, entre otros aspectos. En segundo lugar, se diseñó una matriz para las áreas que comprenden la gestión educativa, concebida a partir de la Guía para 
el Mejoramiento Institucional del Ministerio de Educación Nacional, en la que se relacionan los procesos y componentes de cada área y se asocian con las líneas de investigación de las universidades, teniendo en cuenta la orientación temática de cada línea y sus desarrollos epistemológicos.

Se identificaron 131 líneas de investigación en el campo de la educación, de las cuales 58 fueron categorizadas en las áreas de la gestión educativa, 15 correspondieron al área de gestión directiva, 24 al área de gestión académica, 6 en el área de gestión administrativa y financiera y 17 en gestión de la comunidad. Es de resaltar que 4 líneas de investigación fueron categorizadas en 2 áreas de gestión: 1) en las áreas de gestión directiva y gestión académica: Política, Planificación, Gestión y Evaluación de la Educación Básica (UECO1) -asociada a la gestión directiva y a la gestión académica-, y Evaluación Educativa (UDAO2); y 2), en las áreas de gestión académica y gestión comunitaria: Educación para la Primera Infancia (UDAO3) -tipificada también en el área de gestión directiva y gestión académica-, y Desarrollo Humano y Aprendizaje (UNAMo3).

La investigación se desarrolló desde la perspectiva metodológica de la complementariedad, entendida en los términos de Murcia y Jaramillo, según los cuales, en una investigación que se regula por este enfoque, "las estructuras se van descubriendo, o configurando progresivamente, a medida que se in teractúa con el fenómeno sujeto de estudio" (2000, p. 95). En este sentido, la investigación contempló los siguientes tres círculos investigativos, que se complementaron para comprender las tendencias en la formación investigativa de los programas de formación avanzada en el campo de la gestión educativa en América Latina:

- Círculo 1: Identificación de las líneas de investigación.

- Círculo 2: Caracterización de las tipologías investigativas actuales.
- Círculo 3: Reflexión sobre las tipologías investigativas actuales -con miras a la configuración de escenarios académicos pertinentes en Colombia.

En cada uno de estos círculos se desarrollaron tres momentos investigativos de preconfiguración, configuración y reconfiguración de la realidad (Murcia y Jaramillo, 2000).

Desde el primer momento de preconfiguración de la realidad se logró diseñar una estructura teórica inicial que sirvió para sustentar el objeto de la investigación y sus categorías: gestión directiva, gestión académica, gestión administrativa y financiera y gestión de la comunidad. Adicionalmente, se estableció una estructura inicial empírica que caracterizara las líneas de investigación existentes en el campo de la gestión educativa en América Latina. En el segundo momento, se definieron los elementos teóricos que fundamentaron las categorías centrales de la investigación: gestión educativa y líneas de investigación, al igual que la estructura empírica que la delimitó. Finalmente, en el tercer momento, se confrontaron los resultados de la investigación con los componentes teóricos asociados a la comprensión del objeto de estudio, lo que propició la argumentación discursiva de la investigación y condujo a la reflexión sobre las líneas de investigación exploradas en el campo de la gestión educativa.

\section{Resultados}

A continuación se exponen los hallazgos obtenidos de la sistematización y caracterización de las 58 unidades comprensivas que integran la investigación. Para la identificación de las líneas de investigación de cada universidad en la Tabla 1 se codificaron los nombres de las instituciones de educación superior focalizadas.

\section{Gestión directiva}

La gestión directiva es la primera área asociada a la gestión educativa, de acuerdo con el Ministerio de Educación Nacional, y hace referencia "a la mane- 
Tabla 1. Codificación de universidades

\begin{tabular}{|l|c|}
\hline \multicolumn{1}{|c|}{ Universidad } & Código \\
\hline Pontificia Universidad Católica de Chile & PUCC \\
\hline Universidad Nacional Autónoma de México & UNAM \\
\hline Universidad de Chile & UCH \\
\hline Universidad de Buenos Aires & UBA \\
\hline Universidade de São Paulo & USP \\
\hline Universidade Estadual de Campinas & UEC \\
\hline Universidad Nacional de Colombia & UNC \\
\hline Pontificia Universidad Católica de Valparaíso & PUCV \\
\hline Universidad de los Andes & UDA \\
\hline Universidad de Santiago de Chile & USC \\
\hline Universidade Federal do Rio de Janeiro & UFRJ \\
\hline Universidade Federal do Rio Grade do Sul & UFRG \\
\hline
\end{tabular}

Fuente: elaboración propia.

ra como el establecimiento educativo es orientado" de modo "que el rector o director y su equipo de gestión organicen, desarrollen y evalúen el funcionamiento general de la institución" (MEN, 2008, p. 27). Según Polo y Liñán (2017), es un proceso complejo donde confluyen tensiones administrativas y pedagógicas. Esta área de gestión está integrada por seis procesos: direccionamiento estratégico y horizonte institucional, gestión estratégica, gobierno escolar, cultura institucional, clima escolar y relaciones con el entorno. Dentro de la gestión directiva se identificaron 15 líneas de investigación asociadas a sus procesos y componentes. En cuanto al proceso de direccionamiento estratégico y horizonte institucional, se asociaron tres líneas de investigación: Organización del Trabajo Educativo (USPO1) Teoría de Sistemas y Organizaciones Escolares (PUCVO1) y Las Políticas y las Instituciones Educativas (UFRJo1).

En el proceso de gestión estratégica, por su parte, se articularon 10 líneas de investigación, lo que evidencia la preeminencia de este procesos como constituyente del área de gestión directiva, a saber: Sistemas Educativos Formales y no Formales (UNAM01), Política, Planificación, Gestión y Evalua- ción de la Educación Básica (UECO1), Gestión y Liderazgo Escolar (UNCO1), Liderazgo Escolar (PUCVO2), Liderando el Cambio Sostenido (PUCVo3), Gestión y Políticas Públicas en Educación (UDAo1), Evaluación Educativa (UDAO2), Educación para la Primera Infancia (UDAO3), Evaluación de Carreras e Instituciones (USCO1) y Políticas y Gestión de Procesos Educativos (UFRGO1).

El proceso de gobierno escolar no fue asociado a ninguna línea de investigación, al igual que el de relaciones con el entorno. Por su parte, clima escolar se asoció a la línea Clima Escolar (UCHo4). Finalmente, cultura institucional fue vinculada a las líneas de investigación en cultura escolar: Disciplinamiento, Conflicto y Resistencia (UCHO1) y Cultura, Organización y Educación (USPO2).

Las universidades con mayor número de líneas de investigación en el área de gestión directiva son la Pontificia Universidad Católica de Valparaíso (PUCV) y la Universidad de los Andes (UDA), con 3 líneas de investigación cada una. En lo referente a la Pontificia Universidad Católica de Valparaíso, las líneas de investigación articuladas a la presente área hacen par- 
te del programa de Maestría en Liderazgo y Gestión en Organizaciones Escolares; por otra parte, los programas de formación avanzada de la Universidad de los Andes asociados a la gestión directiva son: Maestría en Educación, Maestría en Educación Matemática y Doctorado en Educación.

\section{Gestión académica}

La gestión académica es definida como "la esencia del trabajo de un establecimiento educativo, pues señala cómo se enfocan sus acciones para lograr que los estudiantes aprendan y desarrollen las competencias necesarias para su desempeño personal, social y profesional" (MEN, 2008, p. 27) y está integrada por cuatro procesos: diseño pedagógico (curricular), prácticas pedagógicas, gestión de aula y seguimiento de aula. Al proceso de diseño pedagógico (curricular) se asociaron 14 líneas de investigación: Medición y Evaluación Educacional (PUCCO3), Teoría y Desarrollo Curricular, Didáctica, Evaluación y Comunicación (UNAMO2), Currículum: Prácticas Pedagógicas, Estrategias de Aprendizaje,
Códigos Curriculares (UCHO2); Currículum, Evaluación y Enseñanza (UECO2); Política, Planificación, Gestión y Evaluación de la Educación Básica (UECO1); Evaluación Educativa (PUCVo4); Educación de las Disciplinas (UDAo4); Evaluación Educativa (UDAO2); Educación para la Primera Infancia (UDAO3); Tópicos de Gestión Académica (USCO2); Currículo, Docencia y Lenguaje (UFRJO2); Arte, Lenguaje y Currículum (UFRGO2), Currículum: Teoría, Planificación e Interacción (PUCVo8), y Tecnologías para la Enseñanza y la Formación (PUCVog).

En el segundo proceso del área de la gestión académica, prácticas pedagógicas, se identificaron 10 líneas de investigación: Enseñanza y Aprendizaje Escolar (PUCCO1); Enseñanza y Aprendizaje en Primera Infancia (PUCCO1); Teoría y Desarrollo Curricular, y Didáctica, Evaluación y Comunicación (UNAMO2); Desarrollo Humano y Aprendizaje (UNAMo3); Enseñanza; Educación y Escuela, y Prácticas de Teorías (USPo3); Prácticas Pedagógicas en la Educación Básica (UECO3); Saber Pedagógico y Profesión Docente (sublínea) (PUCVo5); Docencia Innovativa (USCO3);

Figura 1. Líneas de investigación asociadas a procesos de gestión directiva

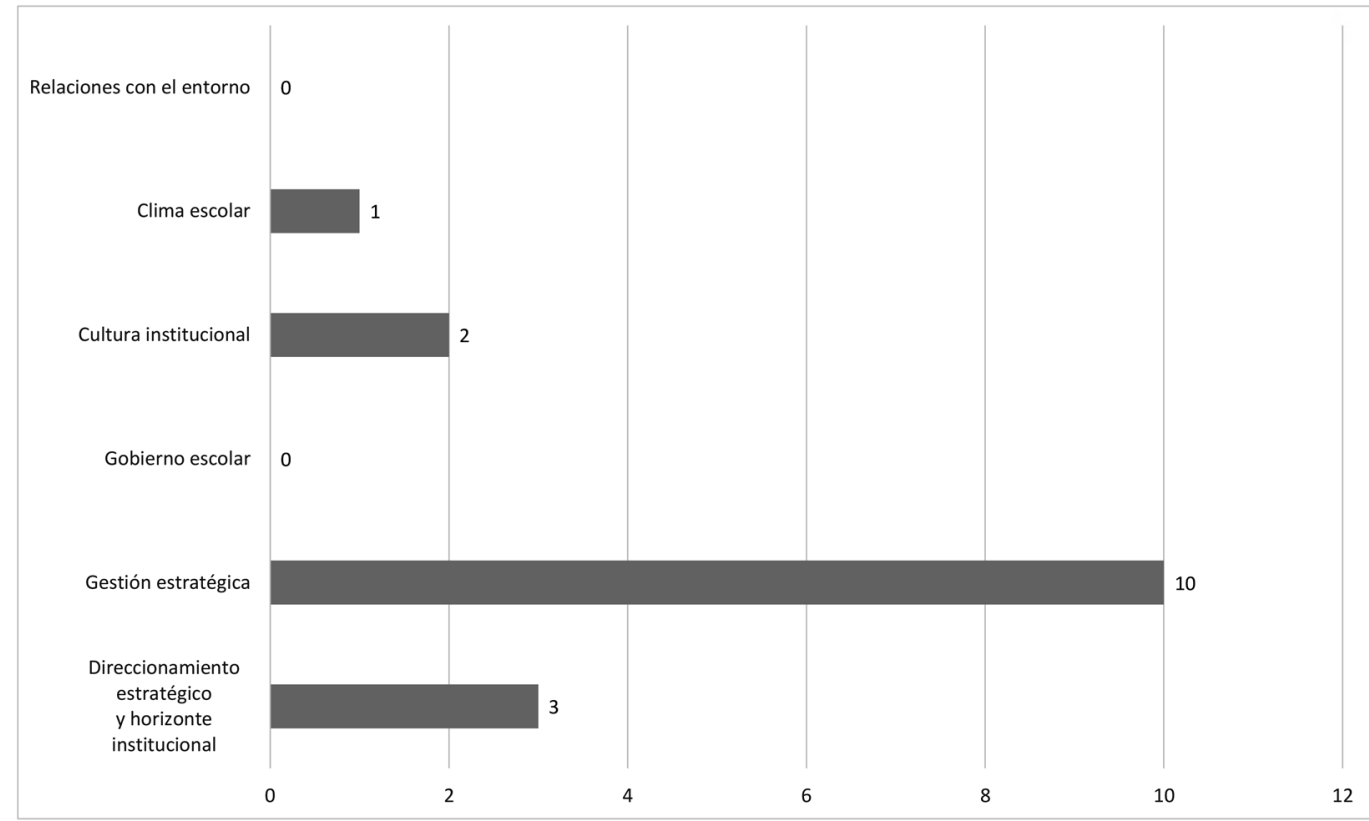

Fuente: elaboración propia. 
Estudios sobre Infancias (UFRGO3) y Construcción de Saberes Pedagógicos (UNAMo4). La gestión del aula se relacionó con 2 líneas de investigación: Enseñanza y Aprendizaje Escolar (PUCCO2) y Docencia (UNAMo5). Por último, en el proceso seguimiento académico se identificó la línea de investigación en Medición y Evaluación Educacional (PUCCO3).

Las Universidades con mayor número de líneas de investigación son: la Pontificia Universidad Católica de Valparaíso (PUCV) y la Universidad Nacional Autónoma de México (UNAM), con 4 líneas de investigación cada una.

\section{Gestión administrativa y financiera}

La gestión administrativa y financiera, definida como "soporte al trabajo institucional" (MEN, 2008, $p$, 27), está constituida por los procesos: apoyo a la gestión académica, administración de la planta física y de los recursos, administración de servicios complementarios, talento humano y apoyo financiero y contable. En esta área de gestión solo se identificaron líneas de investigación en los procesos de apoyo a la gestión académica y talento humano. El primer proceso se asoció con la línea Políticas Educativas y Gestión Académica (UNAMo6) y el segundo con las líneas: Formación de Profesores y Desarrollo Profesional Docente (PUCCO4); La Clase Escolar (UBAO1); Formación de Maestros y Enseñanza (UECo4); Gestión de Recursos Humanos (PUCVo6) y Formación Docente (UDAO5).

En la presente área de gestión no se presentan unidades comprensivas repetidas en las universidades que ofrecen las líneas de investigación, es decir, que cada línea de investigación relacionada con el área de gestión administrativa y financiera pertenece a una universidad diferente. Los programas de formación avanzada asociados a cada línea son: Políticas Educativas y Gestión Académica (UNAMo6) de la Maestría en Pedagogía de la Universidad Nacional Autónoma de México; Formación de Profesores y Desarrollo Profesional Docente (PUCCO4) del Doctorado

Figura 2. Líneas de investigación asociadas a procesos de gestión académica

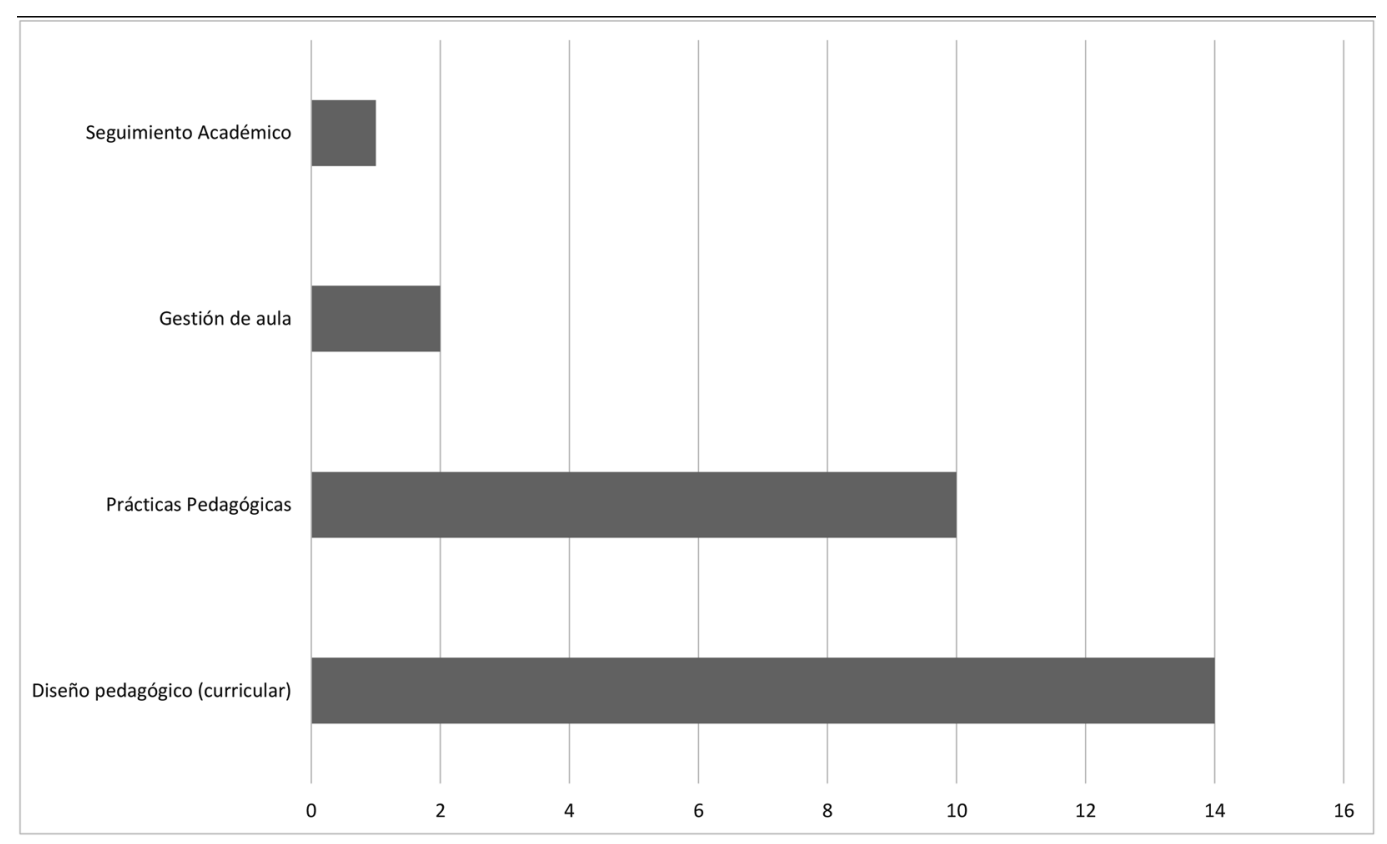

Fuente: elaboración propia. 
en Educación de la Pontificia Universidad Católica de Chile; La Clase Escolar (UBAo1) del Instituto de Investigaciones en Ciencias de la Educación, al que se adscriben todos los programas de posgrado de la Facultad de Filosofía y Letras de la Universidad de Buenos Aires; Formación de Maestros y Enseñanza (UECo4) perteneciente a la Maestría en Educación y al Doctorado en Educación de la Universidad Estadual de Campinas; Gestión de Recursos Humanos (PUCVo6) perteneciente a la Maestría en Liderazgo y Gestión en Organizaciones Escolares de la Pontifica Universidad Católica de Valparaíso, y Formación Docente (UDA05) de los programas de Maestría en Educación, Maestría en Educación Matemática y Doctorado en Educación de la Universidad de los Andes.

\section{Gestión de la comunidad}

La gestión de la comunidad "se encarga de las relaciones de la institución con la comunidad; así como de la participación y la convivencia, la atención educativa a grupos poblacionales con necesidades especiales bajo una perspectiva de inclusión, y la prevención de riesgos" (MEN, 2008, p. 27). Por consiguiente los cuatro procesos del área de gestión comunitaria son: inclusión, proyección a la comunidad, participación y convivencia, y prevención de riesgos. Se encontró que el proceso con mayor número de líneas de investigación es el de inclusión, con 15 líneas: Política, Equidad y Diferencia en Educación, y Liderazgo y Mejora Escolar (PUCCO5); Desarrollo Humano y Aprendizaje (UNAMo3); Sociología de la Educación (UNAMO7); Educación y Diversidad Cultural (UNAMo8); Etnias y Sistema Escolar (UCHo3); Programa de Investigaciones sobre Instituciones Educativas ( UBAO2); Sujetos Sociales y Construcción del Espacio Público en Educación (UBAO3); Educación Especial (USPo4); Ciudadanía, Diversidad e Inclusión (Sublínea) (USPO4); Inclusión, Ética e Interculturalidad (UFRJo3); Tecnologías Digitales en la Educación (UFRGo4); Educación, Culturas y Humanidades (UFRG05); Educación Especial y Procesos Inclusivos ( UFRGo6); Estudios Culturales en Educación (UFRGo7); Diversidad, Derecho a la Educación e Inclusión (PUCV10).

Figura 3. Líneas de investigación asociadas a procesos de gestión administrativa y financiera

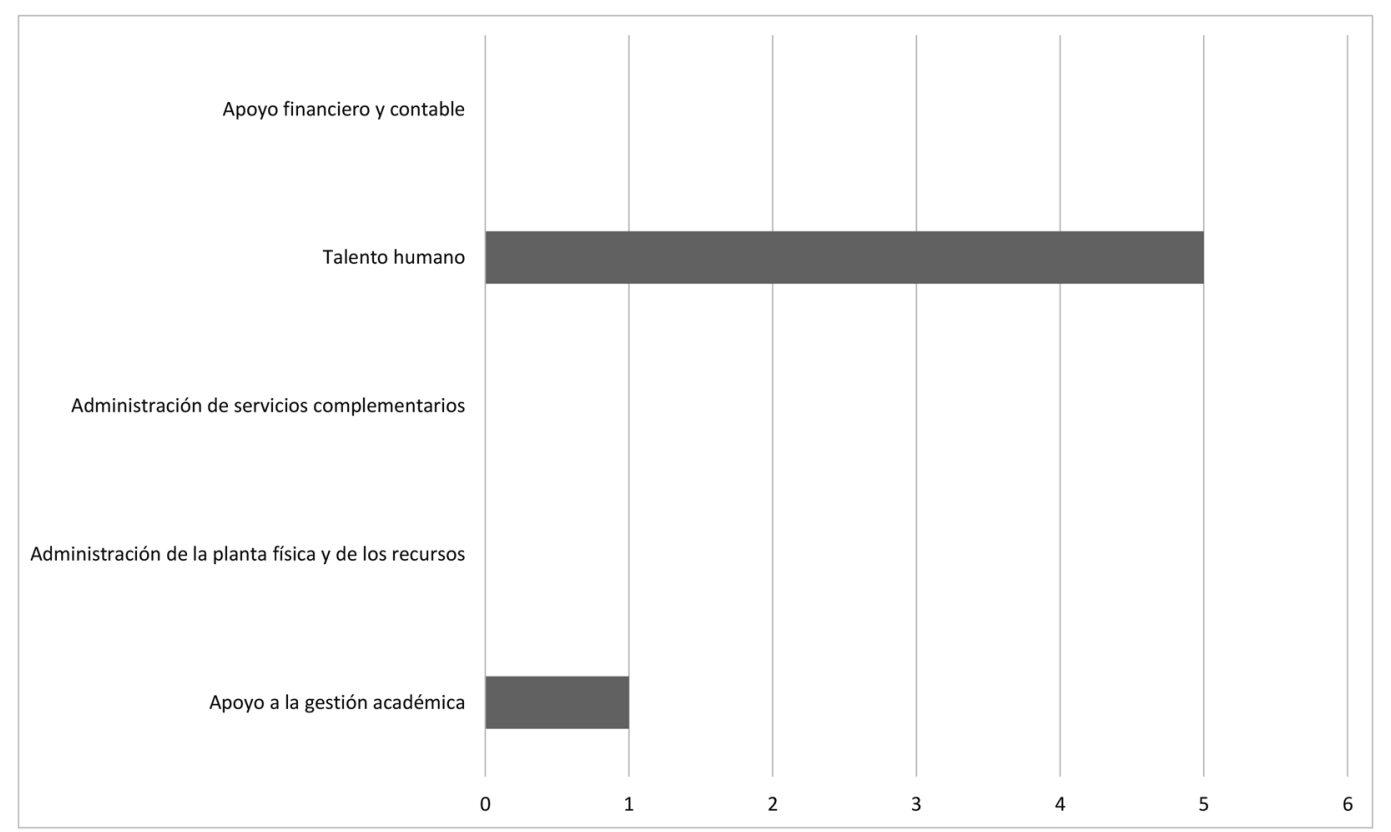

Fuente: elaboración propia. 
Por su parte, en el proceso de proyección a la comunidad se identificaron dos líneas de investigación: Educación Ética, Ciudadanía y Pedagogías Críticas (UDA06) y Educación y TIC (UDA07). Para participación y convivencia y prevención de riesgos no se hallaron líneas de investigación. La universidad con mayor número de líneas de investigación referidas al proceso de gestión de la comunidad es la Universidad Federal do Rio Grande do Sul, con cuatro líneas de investigación.

\section{Discusión}

Los resultados de la investigación permiten ilustrar de manera general las tendencias investigativas en América Latina en el área de la gestión educativa, como aquella disciplina que configura los procesos que tienen lugar dentro de una organización educativa, desde la dirección, pasando por los procesos académicos o de aula, la administración y la gestión comunitaria. Sin embargo, es preciso destacar que, vista la complejidad en la que están inmersas las sociedades actuales, resulta algo cuestionable delimitar todas las dinámicas que se dan dentro de las organizaciones, ya que cada vez más surgen distintos actores que transforman las relaciones organizacionales y amplían las áreas de gestión o procesos institucionales, situación que no es ajena a las instituciones educativas (Palumbo y Manna, 2019), las cuales, por su vocación social, tienen el compromiso de estar en constante resignificación. Ello se debe principalmente a que la organización educativa se constituye en una organización autopoiética, porque "está dada por unidades dinámicas relacionadas en una continua red de interacciones y donde se reconocen la complejidad y la incertidumbre de la realidad. Esto permite a la organización educativa no sólo comprender el medio, sino adecuarse también a él" (Castro, 2001, s.p.). Adicionalmente, ante este panorama se hace fundamental la creación de marcos políticos que fortalezcan la gestión educativa de las instituciones, al igual que líderes educativos (Graffe, 2002; Jedaman, Buaraphan, Pimvichai, Yuenyong y Jeerasombat, 2019) con capacidades y competencias en todas las áreas de gestión, todo esto propiciado por escenarios formativos (Portugal, 2013; Rico, 2016).

Figura 4. Líneas de investigación asociadas a procesos de gestión comunitaria

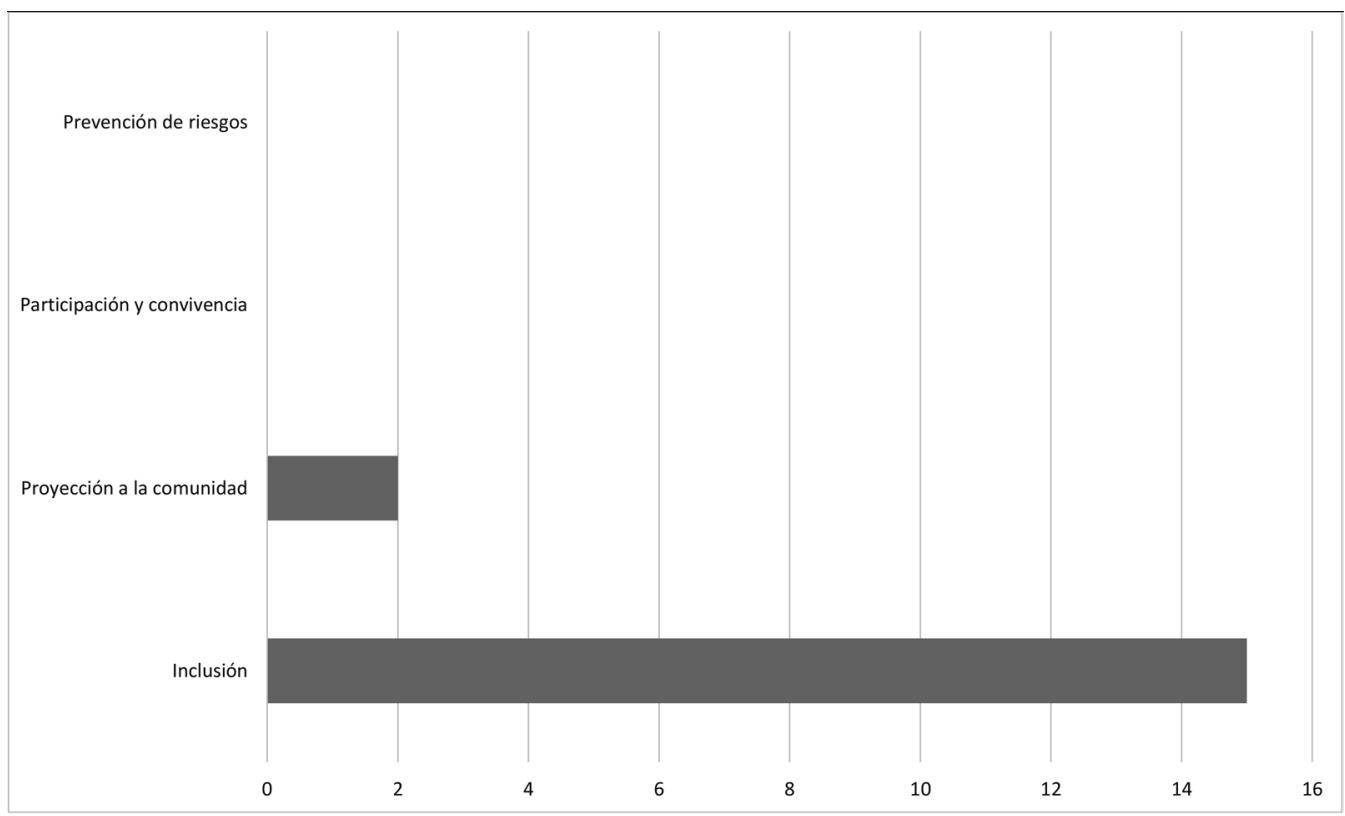

Fuente: elaboración propia 
No obstante, para efectos de esta investigación se hizo necesario explorar la estructura básica de la gestión de las organizaciones educativas. Para ello los autores recurrieron a la estructura planteada por el Ministerio de Educación Nacional como autoridad oficial que rige el accionar educativo en Colombia, debido a que es necesario incentivar procesos investigativos que permitan hacer visibles las estructuras existentes de la gestión educativa, ya que estas "se han vuelto tan universales y perpetuas y, por lo tanto, profundamente arraigadas en la sociedad, que permanecen casi sin ser cuestionadas" (Tarragó y Wilson, 2014, p. 389).

En relación con los resultados, se evidencia la preeminencia de procesos investigativos en el área de gestión académica, específicamente en los componentes de diseño pedagógico y prácticas pedagógicas; seguidamente se encuentra el área de gestión de la comunidad, en la que el mayor número de líneas de investigación fueron asociadas al proceso inclusión, lo que refleja la creciente preocupación por propiciar procesos en los que el sistema educativo se adapte y responda de mejor manera a las necesidades de los estudiantes, situación que se espera que aporte al tan requerido tránsito de escuelas integradoras ${ }^{2}$ a escuelas inclusivas.

En tercer lugar, se encuentra el área de gestión directiva, con el mayor número de líneas de investigación asociadas al proceso gestión estratégica, definido por el MEN (2008) en función de "tener las herramientas esenciales para liderar, articular y coordinar todas las acciones institucionales" ( $p$. 28). En esta área se propone la creación de líneas de investigación en los procesos de gobierno escolar, clima escolar y relaciones con el entorno, las cuales, en concordancia con los resultados, se encuentran

2 La escuela integradora es aquella que exige que el educando se adapte a la oferta educativa existente, por lo que desconoce sus necesidades particulares, concepto ligado a la escuela tradicional. Como contraparte de este tipo de escuela surge la escuela inclusiva, como derecho humano, concebida como aquella "capaz de lograr la adaptación de la oferta educativa a la diversidad del alumnado" (Valcarce, 2011, p. 120). poco exploradas. Se plantea, por tanto, la necesidad de promover dinámicas investigativas encaminadas a investigar aspectos tales como: los elementos de éxito de los consejos directivos, académicos, estudiantiles y de padres de familia; comisión de evaluación y promoción; comités de convivencia; pertenencia y participación en las instituciones; ambiente físico institucional; motivación hacia el aprendizaje; actividades extracurriculares; manejo de conflictos; padres de familia; relaciones con el sector productivo, por citar algunos.

Otro aspecto a destacar con respecto al área de gestión directiva es que la formación avanzada enfocada a los directivos docentes no debe desconocer la necesidad de desarrollar competencias directivas, referidas a las capacidades, habilidades y conocimientos con que cuenta el director escolar para lograr los cambios requeridos en la consolidación de instituciones educativas de calidad (Sánchez, Rúa y Ternera, 2018), ya que, en la mayoría de los casos, la educación impartida se encuadra en aspectos netamente administrativos o de gestión (Rodríguez, 2016), por lo que Santos (2015) resalta la obligación de incentivar la adquisición de conocimientos específicos encaminados a desempeñar la función directiva, toda vez que el liderazgo directivo es uno de las factores determinantes de la calidad educativa en las escuelas (Amanchukwu, Stanley y Ololube, 2015; Yangali, Rodríguez, Vásquez y Chahuara, 2018), cuya formación debe verse reflejada, en primera instancia, en las líneas de investigación de los programas de posgrado.

La última área es la gestión administrativa y financiera, con la más baja cantidad de líneas de investigación, están mayormente asociadas al proceso de talento humano, específicamente en lo relacionado con la formación de docentes y gestión de recursos humanos. Con respecto a los otros procesos, es notoria la falta de actividades investigativas referentes al apoyo a la gestión académica, administración de la planta física y de los recursos, administración de servicios complementarios y apoyo 
financiero y contable. Lo anterior devela la falta de transdiciplinariedad de las investigaciones educativas, al no concebir la necesidad de integrar especialmente a las ciencias administrativas en la comprensión de los fenómenos derivados de la gestión de las organizaciones educativas, lo que fragmenta y reduce la realidad, imposibilita un mejor acercamiento a las problemáticas de la gestión educativa y ocasiona a la vez que se imposibilite la transformación necesaria de los ambientes escolares (Ortiz, 2012). Afirma Santos a propósito que "el conocimiento transdisciplinario se asocia a la dinámica de la multiplicidad de las dimensiones de la realidad y se apoya en el propio conocimiento disciplinario. [...] De ese modo, los conocimientos disciplinarios y transdisciplinarios no se antagonizan, pero se complementan" (2008, p. 75).

En consecuencia, se hace evidente la necesidad de generar procesos investigativos transdisciplinares que incluyan disciplinas como la Administración, la Economía, la Psicológia, la Sociología, la Historia y la Antropología, entre otras que aporten a la mejor comprensión del hecho educativo, todo esto ante una realidad que se complejiza y demanda ser abordada desde diferentes perspectivas, sin descuidar la naturaleza pedagógica de estas organizaciones. Así lo plantean Gibbons et al., quienes aseveran que las nuevas dinámicas de la producción del conocimiento están encaminadas a transcender las estructuras disciplinares y a propiciar procesos transdisciplinares, pues dicha dinámica "se halla fuertemente orientada a la solución de problemas [...]. Su núcleo teórico-metodológico, aunque cruza núcleos disciplinares bien establecidos, se ve impulsado a menudo localmente, y está localmente constituido, por lo que tal núcleo es muy sensible a las nuevas mutaciones locales, dependiendo del contexto de aplicación" (2014, p. 9).

Por otro lado, se concibe la investigación como método fundamental para mejorar los procesos de gestión educativa dentro de las instituciones, propiciando además el aseguramiento de la calidad edu- cativa (Ramírez, García y Cruel, 2017), lo que se logra por medio de la comprensión de los fenómenos sociales que tienen lugar en los entornos escolares. Es por ello que dichos procesos investigativos deben ir articulados a las necesidades de los territorios y de los sujetos que lo conforman, entendiendo a las organizaciones educativas como actores partícipes en la configuración de las realidades comunitarias y sociales, a partir de la integración de sus necesidades a "la construcción del sentido de la educación" (López, 2017, p. 208).

\section{Conclusiones}

Al considerar la educación como un factor clave para el desarrollo de las sociedades, ella se convierte en un elemento central para la reflexión y el desarrollo de estrategias que promuevan el fortalecimiento de su calidad, con miras a lograr mayores interpelaciones en el territorio (Perines y Murillo, 2017). En este sentido, como las organizaciones educativas son nichos complejos donde confluyen aspectos sociales diversos, la gestión de esas complejidades requiere de personas dotadas de competencias humanas y profesionales que respondan a las necesidades organizacionales y educativas. Por lo tanto, la investigación educativa, como herramienta que facilita la comprensión y la transformación de las realidades de las organizaciones educativas, emerge como fundamento para la configuración de procesos organizacionales orientados al logro de la calidad educativa, entendiendo a la organización educativa como una institución que conlleva prácticas pedagógicas en todas sus áreas y procesos (Sánchez, Linero y Martínez, 2014).

En América Latina y especialmente en Colombia, la gestión de las organizaciones educativas ha estado permeada por los discursos administrativos predominantes y se ha distanciado de las intencionalidades teleológicas de las mismas, como consecuencia de la existencia y dominio de modelos empresariales enfocados en los tecnicismos propios de los enfoques de gestión y procedimentales. Por 
tanto, las investigaciones enfocadas en su análisis, comprensión y desarrollos deben estar mediadas por intencionalidades no solamente empíricas, sino también epistemológicas, con miras a la generación de conocimientos académicos y sociales que sustenten dicha práctica. En palabras de Tello, se propone "gestionar la escuela para describir, interpretar e intervenir. Esto nos posiciona ante una conceptualización dinámica de la gestión educativa, en constante cambio según las realidades" (2008, p. 5).

Las apuestas investigativas de los programas de maestría y doctorado en América Latina, expresadas en líneas de investigación, evidencian las tendencias y preocupaciones respecto de los procesos de gestión estratégica y los elementos propios de la diversidad humana que confluyen en una organización educativa. La investigación en el campo de la gestión educativa realizada en las maestrías y doctorados es una opción efectiva para la transformación de las realidades de los territorios y la potenciación de las condiciones de calidad de la educación, considerando que los proyectos de investigación que se desarrollan están enfocados en comprender e intervenir problemáticas que inciden negativamente en los indicadores de calidad y en los ambientes sociales de las instituciones educativas.

Adicionalmente, es importante y necesaria una resignificación del concepto de gestión educativa que subyace a las líneas de investigación, buscando que esta se entienda como una praxis pedagógica que asume a las organizaciones educativas como construcciones sociales, desde la perspectiva de la complejidad organizacional. En este sentido, la gestión educativa demanda espacios que propicien la transformación de sus prácticas en praxis pedagógica; "es decir, en ejercicios conscientes mediados por la reflexión y sustentados en teorías críticas de la educación, que le otorgan nuevos sentidos, hori- zontes y posibilidades a toda actuación social regulada por una finalidad formativa" (Sánchez, 2009, p. 2), sentidos, horizontes y posibilidades que aportan al carácter crítico, de debate y de argumentación teórica en las configuraciones derivadas de los sistemas educativos.

De esta forma, la gestión educativa, considerada a luz de la pedagogía, trae implícita la particularidad de ser una praxis comprensiva y transformadora de las organizaciones y los sistemas educativos, todo ello con el propósito de ampliar el dominio de lo educativo, integrando en él la formación humana y el desarrollo social, lo cual se convierte en un medio para alcanzar lo sustancial, pero a la vez el objetivo más altruista de la praxis de la enseñanza: la formación integral de los educandos. Esto favorece en su esencia a la perspectiva de la sociedad como un entramado diverso, en el que tiene lugar los procesos de realización ontológicos. La gestión educativa, desde la óptica de lo pedagógico, posibilita la resignificación de las prácticas educativas y coadyuva así a la génesis de nuevas prácticas que sean coherentes con las realidades formativas y que contribuyan a la construcción de la deseada calidad educativa.

Por último, la limitación principal que se presentó durante el desarrollo de la investigación fue el acceso a la información, debido a que los datos concernientes a las líneas de investigación fueron recuperados de los sitios web de las universidades focalizadas, los cuales en algunas universidades se presentan de manera limitada. Se espera que esta investigación sirva de antecedente para la identificación de vacíos teóricos en el campo de la gestión educativa, de tal manera que se propicien nuevos desarrollos epistemológicos en esta categoría y se promuevan en Colombia procesos de formación de gestores educativos que están a la vanguardia de las dinámicas internacionales. 


\section{Referencias}

Amanchukwu, R. N., Stanley, G. J. y Ololube, N. P. (2015). A review of leadership theories, principles and styles and their relevance to educational management. Management, 5(1), 6-14. Recuperado de http://article. sapub.org/10.5923.j.mm.20150501.02.html

Ardashkin, I. (2015). Philosophy of Education as a Social Development Factor: World Trends and Prospects for Russia. Procedia - Social and Behavioral Sciences, 166(7), 277-286. DOI: https://doi.org/10.1016/j.sbspro.2014.12.524

Barrios, M. (1990). Criterios y estrategias para la definición de líneas de investigación y prioridades para su desarrollo. Mimeografiado. Caracas: Universidad Pedagógica Experimental Libertador.

Başarı, G., Aktepebaşı, A., Yağcı, E. y Akdağ, Ş. (2017). Postgraduate thesis assessment in educational management supervision and planning. Procedia Computer Science, 120, 887-892. DOI: https://doi.org/10.1016/j. procs.2017.11.322

Botero Chica, C. A. (2009). Cinco tendencias de la gestión educativa. Revista Iberoamericana de Educación, 49(2), 1-11. Recuperado de https://rieoei.org/RIE/article/view/2100

Casassus, J. (2000). Problemas de la gestión educativa en América Latina: la tensión entre los paradigmas de tipo A y el tipo B. Em Aberto, 19(75). Recuperado de http://emaberto.inep.gov.br/index.php/emaberto/ article/view/2167/

Castro Sáez, B. (2001). La organización educativa: una aproximación desde la complejidad. Estudios pedagógicos, (27), 97-110. Recuperado de http://www.redaly c.org/pdf/1735/173513844007.pdf

Cerda, H. (2004). Hacia la construcción de una línea de investigación (Seminario-Taller). Bogotá: Universidad Cooperativa de Colombia.

Connolly, M., James, C. y Fertig, M. (2017). The difference between educational management and educational leadership and the importance of educational responsibility. Educational Management Administration \& Leadership, 2O(20), 1-6. DOI: https://doi.org/10.1177/1741143217745880

Deslauriers, J. (2004). Investigación Cualitativa: Guía Práctica. Rudecolombia. Pereira: Papiro.

Díaz Domínguez, T. y Alemán, P. (2008). La educación como factor de desarrollo. Revista Virtual Universidad Católica del Norte, 23, 1-15. Recuperado de http://revistavirtual.ucn.edu.co/index.php/RevistaUCN/article/view/149

Dumciuviene, D. (2015). The Impact of Education Policy to Country Economic Development. Procedia - Social and Behavioral Sciences, 191(2), 2427-2436. DOI: https://doi.org/10.1016/j.sbspro.2015.04.302

Farfán-Tigre, A., Mero, O. y Sáenz, J. (2016). Consideraciones generales acerca de la gestión educativa. Dominio de las Ciencias, 2(4), 179-19o. Recuperado de https://dominiodelasciencias.com/ojs/index.php/es/article/view/225

Gamage, D. (2006). Professional Development for Leaders and Managers of Self-Governing Schools. Suiza: Springer. 
Gibbons, M., Limoges, C., Nowotny, H., Schwartzman, S., Scott, P.y Trow, M. (2014). La nueva producción del conocimiento. La dinámica de la ciencia y la investigación en las sociedades contemporáneas. Tecnología y Construcción, 28(2), 90-105. Recuperado de http://saber.ucv.ve/ojs/index.php/rev_tc/article/view/8400

González-Acuña, E. L. (2017). Gestión de la identidad pedagógica organizacional: En busca de coherencia y unidad de la intencionalidad formativa. En Vida simbólica en las Organizaciones (pp. 51-92). Colección Diversidad y Complejidad Organizacional en América Latina, No. 1. Perspectivas de análisis. México: Hess.

Graffe, G. J. (2002). Gestión educativa para la transformación de la escuela. Revista de Pedagogía, 23(68), 495-517. Recuperado de http://saber.ucv.ve/bitstream/123456789/5355/1/Graffe\%2C\%20G.\%20 Gesti\%C3\%B3n\%2oeducativa\%20\%2opara\%2ola\%2otransformaci\%C3\%B3n\%2ode\%2ola\%2oescuela.\%20 Revista\%2Ode\%2OPedagog\%C3\%ADa\%20N\%C2\%Bo\%2068\%2O2002.PDF

Hernández, A. (2016). Aproximación teórica a modelo de cambio planeado de gestión organizacional para la innovación educativa desde la Teoría de la Complejidad y Empowerment. Educ@ción en Contexto, 2,182-198.

Jedaman, P., Buaraphan, K., Pimvichai, J., Yuenyong, C. y Jeerasombat, S. (2019). Educational management in transition of science: Policies and strategic leaders for sustainable education 4.0 in the 21st century science classroom. AIP Conference Proceedings, 2081(1). DOI: https://doi.org/10.1063/1.5094020

Kangjuan, Lv, Anyum Y., Siyi, G., Maoguo, W. y Xiaohong, X. (2017). Impacts of educational factors on economic growth in regions of China: a spatial econometric approach. Technological and Economic Development of Economy, 23(6), 827-847. DOI: https://doi.org/10.3846/20294913.2015.1071296

López-Paredes, M. A. (2017). La gestión pedagógica. Apuntes para un estudio necesario. Dominio de las Ciencias, 3(1), 201-215. Recuperado de https://dominiodelasciencias.com/ojs/index.php/es/article/view/384

López-Yáñez, J. (2009). Abriendo la caja negra. Una perspectiva sistémica sobre el cambio en las organizaciones educativas. Revista de Educación, 5, 139-155. Recuperado de http://hdl.handle.net/11441/34071

MEN - Ministerio de Educación Nacional (2008). Guía para el mejoramiento institucional. De la autoevaluación al plan de mejoramiento. Recuperado de https://www.mineducacion.gov.co/1759/w3-article 177745. html? noredirect=1

MEN - Ministerio de Educación Nacional. (2013). Sistema Colombiano de Formación de Educadores y Lineamientos de Política. Recuperado de https://www.mineducacion.gov.co/1621/w3-propertyvalue-48466.html

Morillo, J. P. y González, A. I. (2016). Evaluación teórico-práctica de la Maestría en Ciencias de la Información. Mención: Gerencia del conocimiento. Revista Venezolana de Gerencia, 21(73). Recuperado de http:// produccioncientificaluz.org/index.php/rvg/article/view/21061

Murcia, N. y Jaramillo, L. (2000). La investigación cualitativa. La investigación etnográfica. Armenia: Kinesis.

Ortiz, E. A. (2012). La interdisciplinariedad en las investigaciones educativas. Didasc@lia:Didáctica y Educación, 1, 1-12.

Palumbo, R. y Manna, R. (2019). Making educational organizations able to change: a literature review. International Journal of Educational Management, 33(4), 734-752. DOI: https://doi.org/10.1108/IJEM-02-2018-0051 
Perines, H. y Murillo, F. J. (2017). ¿Cómo mejorar la investigación educativa? Sugerencias de los docentes. Revista de la Educación Superior, 46(181), 89-104. DOI: https://doi.org/10.1016/j.resu.2016.11.003

Polo, M. y Liñán, C. (2017). El proyecto educativo institucional-PEI: Una mirada desde la gestión directiva. En Prácticas y realidades de las organizaciones educativas (pp. 83-117). Colección Diversidad y Complejidad Organizacional en América Latina, No. 1. Perspectivas de análisis. México: Hess.

Portugal, J. (2013). La gestión educativa: Una visión hacia la formación docente. Revista Motricidad y Persona, 12, 33-40.

Ramírez-Solis, C. R., García-Ramírez, E. E. y Cruel-Angulo, J. P. C. (2017). Gestión educativa y desarrollo social. Dominio de las Ciencias, 3(1), 378-39o. Recuperado de http://dominiodelasciencias.com/ojs/index.php/ es/article/view/801

Rico, A. (2016). La gestión educativa: Hacia la optimización de la formación docente en la educación superior en Colombia. Sophia, 12(1), 55-70. Recuperado de http://www.scielo.org.co/pdf/sph/v12n1/v12n1a04.pdf

Rodríguez, Á. (2017a). Las concepciones del liderazgo educativo a partir de las voces de los actores sociales de la gestión educativa en la organización escolar. En Prácticas y realidades de las organizaciones educativas (pp. 223-260). Colección Diversidad y Complejidad Organizacional en América Latina, No. 1. Perspectivas de análisis. México: Hess.

Rodríguez, Á. (2017b). Praxis de liderazgo en la gestión educativa de la organización escolar: Una mirada reflexiva desde los directivos docentes y docentes. En Prácticas y realidades de las organizaciones educativas (pp. 261-299). Colección Diversidad y Complejidad Organizacional en América Latina, No. 1. Perspectivas de análisis. México: Hess.

Rodríguez, E. (2016). Formación para la función directiva escolar en la zona 8. Un análisis a partir de las percepciones de los directores. Yachana, 5(2), 105-117. Recuperado de http://revistas.ulvr.edu.ec/index.php/yachana/article/view/382

Sánchez, J., Linero, D. y Martínez, M. (2014). Ocultamiento del discurso pedagógico frente al discurso administrativo en la gestión de las organizaciones educativas. Clío América, 8(15), 36-46. Recuperado de http:// revistas.unimagdalena.edu.co/index.php/clioamerica/article/view/828

Sánchez, J., Sánchez, I. y Viloria, J. (2016). Problemas y necesidades en la gestión de las organizaciones educativas: implicaciones para la formación avanzada de los docentes y directivos docentes del departamento del Magdalena. En: R. Muñoz, Administración y estudios organizacionales. Complementariedades y contradicciones. Medellín: Red Pilares.

Sánchez, J., Sánchez, I. y Viloria, J. (2017). Expectativas de formación avanzada en el campo de la gestión educativa: una lectura desde las voces de directivos docentes en el departamento del Magdalena, Colombia. En Territorio y organizaciones (pp. 21-59). Diversidad y Complejidad Organizacional en América Latina, No. 1. Perspectivas de análisis. México: Hess.

Sánchez, J. (2009). La resignificación: un método para transformar las prácticas de gestión en las instituciones educativas. Praxis, 5(1), 183-200. Recuperado de http://revistas.unimagdalena.edu.co/index.php/ praxis/article/view/98 
Sánchez, J. (2012). La constitución del objeto de estudio de la Línea de Investigación sobre Administración y Desarrollo de los Sistemas Educativos. Doctorado en Ciencias de la Educación Universidad del Magdalena. Santa Marta: Rudecolombia.

Sánchez, J. O., Rúa, J. C. y Ternera, L. E. (2018). Competencias de los directivos docentes para la transformación de las instituciones educativas en organizaciones escolares inteligentes. Clío América, 12(24). Recuperado de http://revistas.unimagdalena.edu.co/index.php/clioamerica/article/view/2650

Santos, A. (2008). Complexidade e transdisciplinaridade em educação: cinco princípios para resgatar o elo perdido. Revista Brasileira de Educação, 13(37), 71-83.

Santos, M. (2015). Las feromonas de la manzana: El valor educativo de la dirección escolar. Barcelona: Graó.

Tarragó, F. R. y Wilson, A. E. (2014). Educational Management Challenges for the $21^{\text {st }}$ Century. En Key Competencies in the Knowledge Society (pp.389-400). Berlín-Heidelberg: Springer.

Tejada, J. (2000). La educación en el marco de una sociedad global: algunos principios y nuevas exigencias. Profesorado. Revista de Currículum y Formación del Profesorado 4(1), 1-13.

Tello, C. (2008). Gestionar la escuela en Latinoamérica. Gestión educativa, realidad y política. Revista Iberoamericana de Educación, 46(6), 149-156. Recuperado de https://rieoei.org/RIE/article/view/2024

Valcarce, M. (2011). De la escuela integradora a la escuela inclusiva. Innovación Educativa, 21, 119-131. Recuperado de http://hdl.handle.net/10347/6228

Vidal-Ledo, M., Durán-García, F. y Pujal-Victoria, N. (2008). Gestión educativa. Educación Médica Superior, 22(2). Recuperado de http://scielo.sld.cu/scielo.php?script=sci_arttext\&pid=So864-21412008000200012

Yangali, J., Rodríguez, J., Vásquez, M. y Chahuara, J. (2018). La relación de la toma de decisiones y la gestión educativa en docentes gestores de la universidad. Innova. Research Journal, 3(8.1), 60-76. DOI: https://doi. org/10.3389o/innova.v3.n8.1.2018.758 